\title{
Circulating Sclerostin and Irisin Are Related and Interact with Gender to Influence Adiposity in Adults with Prediabetes
}

\author{
Theerawut Klangjareonchai, ${ }_{1}^{1}$ Hataikarn Nimitphong, ${ }^{1,2}$ Sunee Saetung, \\ Nuttapimon Bhirommuang, ${ }^{1}$ Rattanapan Samittarucksa, ${ }^{1}$ Suwannee Chanprasertyothin, ${ }^{1}$ \\ Rattana Sudatip, ${ }^{1}$ and Boonsong Ongphiphadhanakul ${ }^{1,2}$ \\ ${ }^{1}$ Department of Medicine and Research Center, Ramathibodi Hospital, Mahidol University, Rama VI Street, Rajathevi, \\ Bangkok 10400, Thailand \\ ${ }^{2}$ Division of Endocrinology and Metabolism, Department of Medicine, Faculty of Medicine, Ramathibodi Hospital, \\ Mahidol University, Rama 6 Road, Rajathevi, Bangkok 10400, Thailand
}

Correspondence should be addressed to Hataikarn Nimitphong; hataikarnn@hotmail.com

Received 16 April 2014; Revised 29 July 2014; Accepted 17 August 2014; Published 3 September 2014

Academic Editor: Kevin Sinchak

Copyright (C) 2014 Theerawut Klangjareonchai et al. This is an open access article distributed under the Creative Commons Attribution License, which permits unrestricted use, distribution, and reproduction in any medium, provided the original work is properly cited.

\begin{abstract}
Objectives. Sclerostin, an osteocyte-specific protein, has been found to be related to adiposity and glucose metabolism. Irisin, a myokine, can affect browning of white fat and influence glucose and energy homeostasis. Taken together, this suggests a probable network among fat, bone, and muscle that may influence health outcomes. The aims of this study were to investigate the relationship of circulating sclerostin and irisin and their association with adiposity (assessed by body mass index (BMI)). Materials/Methods. A cross-sectional study included 98 adults with impaired fasting glucose and/or impaired glucose tolerance. 75 gm OGTT was performed in all subjects. Fasting plasma samples were obtained for glycated hemoglobin, calcium, creatinine, serum sclerostin and irisin. Results. Circulating irisin and sclerostin were highly correlated $(r=-0.4 ; P<0.001)$. After controlling for age, gender, and $\mathrm{BMI}$, irisin was significantly related to sclerostin $(P<0.001)$. Multivariate linear regression analysis demonstrated that circulating sclerostin $(\beta=-0.45 ; P<0.05)$ and irisin $(\beta=-0.46 ; P<0.05)$ were negatively associated with BMI, independent of age in males. In females, no relationship of sclerostin or irisin to BMI was found. Conclusions. Circulating irisin and sclerostin are highly related. Interventions targeting irisin could affect sclerostin and vice versa.
\end{abstract}

\section{Introduction}

Multipotential mesenchymal stem cells (MSCs) are able to differentiate into adipocytes, myocytes, and osteoblasts. Canonical Wnt or Wnt $/ \beta$-catenin signaling regulates MSC differentiation [1]. Activation of canonical Wnt signaling promotes differentiation of MSCs into myoblasts and osteoblasts but inhibits differentiation into preadipocytes [2]. Canonical Wnt signaling organizes the balance among adipogenesis, myogenesis, and osteogenesis [2].

Sclerostin is produced by osteocytes and inhibits the Wnt signaling pathway, which in turn leads to the inhibition of osteoblast differentiation and bone formation [3]. Moreover, Wnt signaling blocks adipogenesis through suppression of
CCAAT/enhancer-binding protein- $\alpha(\mathrm{CEBP} \alpha)$ and peroxisome proliferator-activated receptor- $\gamma(\operatorname{PPAR} \gamma)$ and blocks the thermogenic program through suppression of PPAR $\gamma$ coactivator-1 $\alpha$ (PGC-1 $\alpha)$ [2]. Consequently, sclerostin stimulates adipogenesis or fat production. In humans, a positive correlation has been reported between circulating sclerostin and the percentages of abdominal, gynoid fat [4], and fat mass [5]. In addition, type 2 diabetic subjects had higher circulating sclerostin than in nondiabetic control subjects $[6$, 7]. Sclerostin is also associated with the duration of type 2 diabetes mellitus (T2DM) and the level of glycated hemoglobin (HbAlc) in T2DM patients [8]. To date, there have been reports of positive correlations between sclerostin and male gender, age, body mass index (BMI), and T2DM and negative 
correlations between sclerostin and estrogen replacement, mechanical unloading, and intermittent parathyroid hormone (PTH) therapy [4, 8-12].

Irisin is a recently identified myokine released into the blood circulation during exercise [13]. Irisin is secreted in response to PGC- $1 \alpha$ activation; this hormone induces the transformation of white fat cells into beige fat cells. This type of fat functions, as brown fat, increases total body energy expenditure, reduces body weight, and increases insulin sensitivity $[13,14]$. In humans, existing data of irisin levels in relation to obesity, diabetes, and metabolic syndrome are inconclusive [15]. For examples, an epidemiologic study demonstrated that circulating irisin levels were lower in nondiabetic, overweight, and obese subjects compared to lean subjects [16]. In addition, with comparable BMI, subjects with T2DM had lower levels of circulating irisin than controls [16]. On the contrary, there was a trend of positive association between circulating irisin and BMI in a study of healthy women $[17,18]$. Nonetheless, this association disappeared after adjusting for potential confounders [17]. Other existing data have demonstrated positive correlations between irisin and estradiol level, muscle mass, and insulin sensitivity $[16,17]$. On the other hand, older age and fat mass were negatively correlated with irisin $[16,17]$. Taken together, this suggests a crosstalk among bone and fat though sclerostin and among muscle and fat through irisin. However, no data exists regarding a direct relationship between sclerostin and irisin. Therefore, the aim of the present study was to investigate the relationship of circulating irisin and sclerostin and their association with adiposity as assessed by BMI in adults at high risk of T2DM.

\section{Materials and Methods}

2.1. Study Population. This cross-sectional study was conducted at Ramathibodi Hospital, Mahidol University, Bangkok, Thailand. Two hundred and forty healthy volunteers who aged $35-80$ years were recruited by advertisement for the screening of type 2 diabetes from July 2012 to March 2013. A $75 \mathrm{~g}$ oral glucose tolerance test (OGTT) was performed in the morning after an $8 \mathrm{~h}$ overnight fast to recruit subjects with impaired fasting glucose (IFG) and/or impaired glucose tolerance (IGT) according to American Diabetes Association criteria [19]. There were 98 subjects with IFG and/or IGT included in this study. Other inclusion criteria were adults with normal renal function, hepatic function, and calcium level. Exclusion criteria were known diseases affecting bone (hyperparathyroidism, hypercortisolism, renal bone disease, and chronic liver disease) and previous or current treatment with drugs affecting bone metabolism (calcium supplements, vitamin D supplements, selective estrogen receptor modulators, estrogen therapy, calcitonin, antiresorptive therapy, thiazides, glucocorticoids, and anticonvulsants). The ethical review board of Ramathibodi Hospital, Mahidol University, approved this study; all participants provided written informed consent.
2.2. Clinical Evaluation. The height of each subject was measured to the nearest $0.1 \mathrm{~cm}$ using a stadiometer. Body weight was measured using a digital weighing scale (Soehnle 7755; Nassau, Germany). Body mass index (BMI) was calculated by the Quetelet formula: weight in kilograms divided by the square of height in meters $\left(\mathrm{kg} / \mathrm{m}^{2}\right)$. Waist circumference (WC) was measured at the umbilical level.

2.3. Laboratory Assays. Samples of venous blood were taken in the morning after fasting overnight. Serum was stored at $-80^{\circ} \mathrm{C}$ until examination. Fasting plasma glucose (FPG), $2 \mathrm{~h}$ plasma glucose ( $2 \mathrm{hPG}$ ), glycated hemoglobin (HbAlc), calcium and creatinine were measured using standard automated laboratory techniques.

Serum sclerostin was measured using a quantitative ELISA developed by Biomedica (Vienna, Austria). The intraassay variability was $5 \%$ and the interassay variability was $3 \%$. Serum irisin was measured using a quantitative ELISA developed by AdipoGen (Incheon, South Korea). The expected range in human serum according to the manufacture is $0.2-2 \mu \mathrm{g} / \mathrm{mL}$. There is no report of crossing reactivity with molecule other than irisin. The intra-assay variability was $6.7 \%$, and the interassay variability was $8 \%$.

2.4. Statistical Analysis. Variables were presented as mean \pm standard error of mean (SEM), unless stated otherwise. The Kolmogorov-Smirnov test was used to assess the normality of distribution of continuous variables. All parameters were in normal distribution. Differences between males and females were determined by unpaired Student's $t$-test. Pearson's correlation coefficients described associations between continuous variables. Multivariate linear regression analyses of covariance were used to evaluate the influence of independent variables on serum sclerostin levels or BMI. A $P$ value $<0.05$ was considered statistically significant.

\section{Results}

3.1. Baseline Characteristics of the Study Population. Table 1 shows the clinical characteristics and biochemical parameters of the study population. There were 25 males and 73 females with a mean age of 60 years. When comparing males and females, there were no differences in age, BMI, WC, FPG, 2 hPG, and HbAlc. Circulating irisin was found to be higher in females than in males $(3.08 \pm 0.07$ versus $2.67 \pm$ $0.12 \mu \mathrm{g} / \mathrm{mL} ; t=2.9 ; \mathrm{df}=96 ; P<0.01)$. On the other hand, circulating sclerostin tended to be higher in males $(83.38 \pm$ 5.59 versus $70.52 \pm 3.49 \mathrm{pmol} / \mathrm{L} ; t=1.9 ; \mathrm{df}=96 ; P=0.06$ ).

3.2. Relationship between Sclerostin and Irisin Levels. Circulating sclerostin and irisin were highly correlated $(r=-0.4$; $P<0.001$ ) (Figure 1). Table 2 shows the correlation matrices between sclerostin, irisin, and other relevant variables in males and females. Sclerostin and irisin are negatively correlated in both males (Table 2(a)) and females (Table 2(b)). Sclerostin increased with advancing age only in females (Table 2(b)). After controlling for age, gender, and BMI, irisin was still significantly related to sclerostin, as shown in Table 3. 
TABLE 1: Clinical characteristics of the study population according to gender.

\begin{tabular}{lccc}
\hline & Males $(n=25)$ & Females $(n=73)$ & $P$ value \\
\hline Age $($ years $)$ & $61.08 \pm 2.49$ & $58.37 \pm 1.03$ & 0.32 \\
Body weight $(\mathrm{kg})$ & $74.02 \pm 2.73$ & $66.24 \pm 1.42$ & 0.02 \\
Height $(\mathrm{cm})$ & $164.51 \pm 1.10$ & $152.42 \pm 0.71$ & $<0.001$ \\
BMI $\left(\mathrm{kg} / \mathrm{m}^{2}\right)$ & $27.25 \pm 0.83$ & $28.48 \pm 0.60$ & 0.22 \\
WC $(\mathrm{cm})$ & $95.21 \pm 1.99$ & $96.03 \pm 1.37$ & 0.74 \\
FPG $(\mathrm{mmol} / \mathrm{L})$ & $5.83 \pm 0.11$ & $5.80 \pm 0.06$ & 0.78 \\
2hPG $(\mathrm{mmol} / \mathrm{L})$ & $8.75 \pm 0.16$ & $8.71 \pm 0.17$ & 0.90 \\
HbAlc $(\%)$ & $6.09 \pm 0.06$ & $6.04 \pm 0.04$ & 0.53 \\
Irisin $(\mu \mathrm{g} / \mathrm{mL})$ & $2.67 \pm 0.12$ & $3.08 \pm 0.07$ & $<0.01$ \\
Sclerostin $(\mathrm{pmol} / \mathrm{L})$ & $83.38 \pm 5.59$ & $70.52 \pm 3.49$ & 0.06 \\
\hline
\end{tabular}

Data is expressed as mean \pm SEM.

BMI: body mass index, WC: waist circumference, FPG: fasting plasma glucose, 2hPG: $2 \mathrm{~h}$ plasma glucose, and HbAlc: glycated hemoglobin.

TABle 2: (a) Correlation matrix among sclerostin, irisin, age, BMI, and WC in males with prediabetes $(n=25)$. (b) Correlation matrix among sclerostin, irisin, age, BMI, and WC in females with prediabetes $(n=73)$.

(a)

\begin{tabular}{lccccc}
\hline & Sclerostin & Irisin & Age & BMI & WC \\
\hline Sclerostin & 1 & & & & \\
Irisin & -0.59 & 1 & & & \\
& $P<0.01$ & & & & \\
Age & 0.10 & -0.17 & 1 & & \\
& $P=0.63$ & $P=0.43$ & & & \\
BMI & -0.24 & -0.09 & -0.55 & 1 & \\
& $P=0.26$ & $P=0.66$ & $P<0.01$ & & 1 \\
WC & -0.23 & -0.08 & -0.46 & 0.90 & \\
& $P=0.27$ & $P=0.70$ & $P<0.05$ & $P<0.001$ & \\
\hline
\end{tabular}

BMI: body mass index, WC: waist circumference.

(b)

\begin{tabular}{lccccc}
\hline & Sclerostin & Irisin & Age & BMI & WC \\
\hline Sclerostin & 1 & & & & \\
Irisin & -0.29 & 1 & & & \\
& $P<0.05$ & & & & \\
Age & 0.23 & -0.17 & 1 & & \\
& $P<0.05$ & $P=0.15$ & & & \\
BMI & -0.18 & 0.07 & -0.19 & 1 & 1 \\
& $P=0.13$ & $P=0.55$ & $P=0.10$ & & \\
WC & -0.05 & 0.02 & -0.08 & 0.86 & 1 \\
& $P=0.68$ & $P=0.85$ & $P=0.53$ & $P<0.001$ & \\
\hline
\end{tabular}

BMI: body mass index, WC: waist circumference.

3.3. Multivariate Models including BMI and Serum Sclerostin, Irisin and Age. Multivariate linear regression analysis demonstrated that circulating sclerostin $(\beta=-0.45 ; P<$ $0.05)$ and irisin $(\beta=-0.46, P<0.05)$ were negatively associated with BMI, independent of age in males (Table $4(\mathrm{a})$ ).

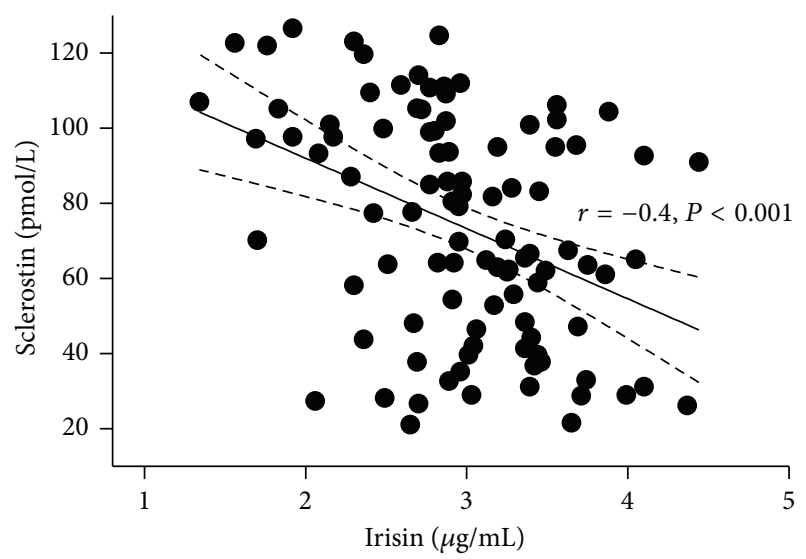

FIGURE 1: Relationship between circulating sclerostin and irisin in the study population $(n=98)$.

TABLE 3: Multivariate linear regression model evaluating the association of sclerostin with irisin after controlling for age, gender, and BMI $(n=98)$.

\begin{tabular}{lcc}
\hline & $\begin{array}{c}\text { Standardized coefficient } \\
(\beta)\end{array}$ & $P$ value \\
\hline Irisin & -0.35 & 0.001 \\
Age & 0.09 & 0.39 \\
Gender & -0.06 & 0.52 \\
$(1=$ male, $2=$ female $)$ & -0.15 & 0.12 \\
BMI & & \\
\hline
\end{tabular}

BMI: body mass index.

TABLE 4: (a) Correlation between BMI and age, sclerostin and irisin in male adults with prediabetes $(n=25)$ by multivariate analysis. (b) Correlation between BMI and age, sclerostin and irisin in female adults with prediabetes $(n=73)$ by multivariate analysis.

(a)

\begin{tabular}{lcccccc}
\hline & \multicolumn{2}{c}{ Model 1 } & \multicolumn{2}{c}{ Model 2 } & \multicolumn{2}{c}{ Model 3 } \\
& $\beta$ & $P$ value & $\beta$ & $P$ value & $\beta$ & $P$ value \\
\hline Age & -0.55 & $<0.01$ & -0.53 & $<0.01$ & -0.58 & $<0.01$ \\
Sclerostin & & & -0.18 & 0.31 & -0.45 & $<0.05$ \\
Irisin & & & & & -0.46 & $<0.05$ \\
\hline
\end{tabular}

Model 1: independent variable: age.

Model 2: independent variables: age, sclerostin.

Model 3: independent variables: age, sclerostin, and irisin.

(b)

\begin{tabular}{lcccccc}
\hline & \multicolumn{2}{c}{ Model 1 } & \multicolumn{2}{c}{ Model 2 } & \multicolumn{2}{c}{ Model 3 } \\
& $\beta$ & $P$ value & $\beta$ & $P$ value & $\beta$ & $P$ value \\
\hline Age & -0.19 & 0.11 & -0.16 & 0.19 & -0.16 & 0.19 \\
Sclerostin & & & -0.14 & 0.24 & -0.14 & 0.26 \\
Irisin & & & & & 0.001 & 0.99 \\
\hline
\end{tabular}

Model 1: independent variable: age.

Model 2: independent variables: age, sclerostin.

Model 3: independent variables: age, sclerostin, and irisin.

In females, no relationship of sclerostin or irisin to BMI was found (Table 4(b)). 


\section{Discussion}

The conventional view of the muscle-bone relationship is that skeletal muscle exerts physical loading on the skeleton and that bone provides an attachment site for skeletal muscle. Beyond that, the knowledge regarding a likely biochemical crosstalk between skeletal muscle and bone is limited [20]. Recently it has been reported that beige fat is anabolic to bone [21]. A study of transgenic mice with the transcription factor forkhead box C2 (FoxC2) that promotes brown fat development indicated that inducible beige fat downregulates the expression of sclerostin. It is likely that the negative association between irisin and sclerostin may be mediated through the influence of irisin on the stimulation of the browning of adipocytes [21]. To our knowledge, the present study is the first report that reveals a correlation of serum sclerostin level with serum irisin. Sclerostin plays a role in bone and fat cell formation $[2,3]$, while irisin plays a well-established role in beige fat cell formation [13]. Taken together, this suggests an interrelationship among fat, bone, and muscle. It is of note that bone, adipose tissue, and skeletal muscle are tissues responsible for high-burden diseases associated with aging, such as osteoporosis, obesity, and sarcopenia. These disorders often occur concurrently and contribute to increases in morbidity and mortality as well as impaired quality of life in the elderly. Together with the results in the present study, it appears that these tissues can often affect one another directly or indirectly through their secretory products. Because of these interacting links of their secretory products among bone, adipose tissue, and skeletal muscle, it complicates the effort to identify the secretory products which are directly causal from clinical study data. Identifying links among secretory products and discriminating those that are direct will enhance our understanding of the interactions and will likely bring about therapeutic measures with fewer adverse effects on other tissues and with simultaneous benefits for more than a single disease. Further more robust studies are needed to systematically identify these interacting links and their causal relationships.

We also demonstrated in this study that age, irisin, and sclerostin were independently associated with adiposity (as assessed by BMI) only in males. Both irisin and sclerostin have been separately reported to be associated with adiposity $[4,16]$. Previous studies have shown that irisin mRNA is decreased in both adipose tissue and muscle in obese subjects [16]. There are conflicting data about the relationship between irisin and adiposity as there were reports of both negative [16] and positive correlations $[17,18]$. In our study, the negative correlation between irisin and BMI when controlling for age and irisin in males subjects is similar to the findings in the study of Moreno-Navarrete et al. [16]. Additional studies with the larger sample size are needed to elucidate the physiological role of irisin in obesity. On the other hand, sclerostin was found to be positively related to BMI or other measures of adiposity in studies of nondiabetic subjects $[4,5,22]$. However, no significant relationship between sclerostin and BMI was detected in a study of subjects with type 2 diabetes [8]. Likewise, no relationship between sclerostin and BMI was found in our prediabetes study population. It thus appears that glucose tolerance status may influence the relationship between sclerostin and adiposity. However, when controlling for irisin, there was a significant relationship between sclerostin and BMI in males, but the direction of association was opposite to that reported in nondiabetic subjects. This may be suggestive of the existence of the influence of irisin on BMI, or vice versa, in subjects with prediabetes, which should be taken into account when trying to determine the unconfounded relationship between sclerostin and adiposity in abnormal glucose tolerance status.

In the present study, we found a sexual dimorphism in terms of BMI in relation to serum irisin and sclerostin of which the underlying basis is not entirely clear. However, it is well established that sexual dimorphism exists for body composition. In general, adult males have higher lean mass and bone mass, but a lower fat mass than females after corrected for body size [23]. Such difference is likely to be attributable to sex steroid hormones and probably their interaction [24]. Moreover, estradiol level may influence serum sclerostin and irisin $[17,25]$. Based on the complex interrelationship among sex steroid hormones, body composition, and irisin, as well as sclerostin, the relationship between BMI and sclerostin as well as irisin could be different according to gender, as shown in our study.

Our data confirmed previously have shown positive relationships between age and sclerostin levels in both genders. Previous studies reported that at any given total body bone mineral content and presumably an equivalent number of osteocytes, serum sclerostin levels were higher in the elderly compared to young subjects. This is suggesting that with aging, there is an increased sclerostin production by individual osteocytes. Nonetheless, the reduction in sclerostin clearance with aging could not be entirely excluded $[8,12]$. Moreover, our study also confirmed previous findings that males have higher serum sclerostin levels and lower serum irisin levels than females; this is in accordance with recent studies showing that sclerostin and irisin are partly regulated by estrogen [17, 25-27].

The present study has a number of limitations. Our study shows only association. The causal relationship between sclerostin and irisin still remains to be determined. The sample is relatively small and may affect the statistical power of our study. It could be a concern that circulating sclerostin may not reflect sclerostin levels in bone. However, bone marrow plasma and peripheral serum sclerostin concentrations are strongly correlated [28]. This suggests that peripheral serum sclerostin may be a good surrogate of sclerostin secreted from osteocytes. Finally, we did not have data about the physical activity indexes of the subjects enrolled. This may be a weakness of the study since both sclerostin and irisin are influenced by physical activity.

In conclusion, this study demonstrates that serum sclerostin is highly correlated with serum irisin in adults with prediabetes, independent of age, gender, and BMI. Also, circulating sclerostin and irisin are negatively associated with BMI, independent of age in males but not females. We postulate that this relationship might be the language of the crosstalk between fat, bone, and muscle. Additional 
prospective longitudinal studies are warranted to evaluate the effect of serum irisin on serum sclerostin, BMI, and fat mass.

$\begin{array}{ll}\text { Abbreviations } \\ \text { BMI: } & \begin{array}{l}\text { Body mass index (weight in kilograms } \\ \text { divided by the square of height in meters } \\ \left.\left(\mathrm{kg} / \mathrm{m}^{2}\right)\right)\end{array} \\ \text { 75 gm OGTT: } & 75 \text { grams oral glucose tolerance } \\ \text { HbAlc: } & \text { Glycated hemoglobin } \\ \text { MSCs: } & \text { Multipotential mesenchymal stem cells } \\ \text { CEBP } \alpha: & \text { CCAAT/enhancer-binding protein- } \alpha \\ \text { PPAR } \gamma: & \text { Peroxisome proliferator-activated } \\ & \text { receptor- } \gamma \\ \text { PGC- } 1 \alpha: & \text { PPAR } \gamma \text { coactivator-1 } \alpha \\ \text { T2DM: } & \text { Type } 2 \text { diabetes mellitus } \\ \text { PTH: } & \text { Parathyroid hormone } \\ \text { IFG: } & \text { Impaired fasting glucose } \\ \text { IGT: } & \text { Impaired glucose tolerance } \\ \text { WC: } & \text { Waist circumference } \\ \text { FPG: } & \text { Fasting plasma glucose } \\ 2 \text { hPG: } & \text { 2h plasma glucose } \\ \text { ELISA: } & \text { Enzyme-linked immunosorbent assay } \\ \text { SEM: } & \text { Standard error of mean } \\ \text { FoxC2: } & \text { Transcription factor forkhead box C2. }\end{array}$

\section{Conflict of Interests}

The authors declare that there is no conflict of interests that could prejudice the impartiality of the research reported.

\section{Authors' Contribution}

Theerawut Klangjareonchai, Hataikarn Nimitphong, and Boonsong Ongphiphadhanakul conceived the study and participated in its design and coordination, performed the statistical analysis, and helped in drafting the paper. Sunee Saetung, Nuttapimon Bhirommuang, Rattanapan Samittarucksa, and Rattana Sudatip participated as study coordinators. Suwannee Chanprasertyothin carried out the biochemical measurement.

\section{Acknowledgments}

The authors thank all the staff at the clinical sites as well as the study subjects for their participation in this study. This work was supported by Mahidol University and the National Science and Technology Development Agency of Thailand.

\section{References}

[1] C. Y. Logan and R. Nusse, "The Wnt signaling pathway in development and disease," Annual Review of Cell and Developmental Biology, vol. 20, pp. 781-810, 2004.

[2] C. Christodoulides, C. Lagathu, J. K. Sethi, and A. Vidal-Puig, "Adipogenesis and WNT signalling," Trends in Endocrinology and Metabolism, vol. 20, no. 1, pp. 16-24, 2009.
[3] M. J. Moester, S. E. Papapoulos, C. W. Löwik, and R. L. van Bezooijen, "Sclerostin: current knowledge and future perspectives," Calcified Tissue International, vol. 87, no. 2, pp. 99-107, 2010.

[4] T. Urano, M. Shiraki, Y. Ouchi, and S. Inoue, "Association of circulating sclerostin levels with fat mass and metabolic disease-related markers in Japanese postmenopausal women," Journal of Clinical Endocrinology and Metabolism, vol. 97, no. 8, pp. E1473-E1477, 2012.

[5] Z. Sheng, D. Tong, Y. Ou et al., "Serum sclerostin levels were positively correlated with fat mass and bone mineral density in Central South Chinese postmenopausal women," Clinical Endocrinology, vol. 76, no. 6, pp. 797-801, 2012.

[6] L. Gennari, D. Merlotti, R. Valenti et al., "Circulating Sclerostin levels and bone turnover in type 1 and type 2 diabetes," Journal of Clinical Endocrinology and Metabolism, vol. 97, no. 5, pp. 17371744, 2012.

[7] M.-S. M. Ardawi, D. H. Akhbar, A. AlShaikh et al., "Increased serum sclerostin and decreased serum IGF-1 are associated with vertebral fractures among postmenopausal women with type-2 diabetes," Bone, vol. 56, no. 2, pp. 355-362, 2013.

[8] A. García-Martín, P. Rozas-Moreno, R. Reyes-García et al., "Circulating levels of sclerostin are increased in patients with type 2 diabetes mellitus," The Journal of Clinical Endocrinology \& Metabolism, vol. 97, no. 1, pp. 234-241, 2012.

[9] A. H. van Lierop, J. E. Witteveen, N. A. T. Hamdy, and S. E. Papapoulos, "Patients with primary hyperparathyroidism have lower circulating sclerostin levels than euparathyroid controls," European Journal of Endocrinology, vol. 163, no. 5, pp. 833-837, 2010.

[10] A. Gaudio, P. Pennisi, C. Bratengeier et al., "Increased sclerostin serum levels associated with bone formation and resorption markers in patients with immobilization-induced bone loss," Journal of Clinical Endocrinology and Metabolism, vol. 95, no. 5, pp. 2248-2253, 2010.

[11] M.-S. M. Ardawi, H. A. Al-Kadi, A. A. Rouzi, and M. H. Qari, "Determinants of serum sclerostin in healthy pre- and postmenopausal women," Journal of Bone and Mineral Research, vol. 26, no. 12, pp. 2812-2822, 2011.

[12] U. I. Mödder, K. A. Hoey, S. Amin et al., "Relation of age, gender, and bone mass to circulating sclerostin levels in women and men," Journal of Bone and Mineral Research, vol. 26, no. 2, pp. 373-379, 2011.

[13] P. Boström, J. Wu, M. P. Jedrychowski et al., "A PGC1- $\alpha$ dependent myokine that drives brown-fat-like development of white fat and thermogenesis," Nature, vol. 481, no. 7382, pp. 463-468, 2012.

[14] B. M. Spiegelman, "Banting lecture 2012: regulation of adipogenesis: toward new therapeutics for metabolic disease," Diabetes, vol. 62, no. 6, pp. 1774-1782, 2013.

[15] M. G. Novelle, C. Contreras, A. Romero-Picó, M. López, and C. Diéguez, "Irisin, two years later," International Journal of Endocrinology, vol. 2013, Article ID 746281, 8 pages, 2013.

[16] J. M. Moreno-Navarrete, F. Ortega, M. Serrano et al., "Irisin is expressed and produced by human muscle and adipose tissue in association with obesity and insulin resistance," The Journal of Clinical Endocrinology \& Metabolism, vol. 98, no. 4, pp. E769E778, 2013.

[17] J. Y. Huh, G. Panagiotou, V. Mougios et al., "FNDC5 and irisin in humans: I. Predictors of circulating concentrations in serum 
and plasma and II. mRNA expression and circulating concentrations in response to weight loss and exercise," Metabolism: Clinical and Experimental, vol. 61, no. 12, pp. 1725-1738, 2012.

[18] A. B. Crujeiras, M. Pardo, R. R. Arturo et al., "Longitudinal variation of circulating irisin after an energy restriction-induced weight loss and following weight regain in obese men and women," American Journal of Human Biology, vol. 26, no. 2, pp. 198-207, 2014.

[19] American Diabetes Association, "Standards of medical care in diabetes-2013,” Diabetes Care, vol. 36, supplement 1, pp. S11S66, 2013.

[20] E. Schoenau, "From mechanostat theory to development of the "functional muscle-bone-unit"', Journal of Musculoskeletal Neuronal Interactions, vol. 5, no. 3, pp. 232-238, 2005.

[21] S. Rahman, Y. Lu, P. J. Czernik, C. J. Rosen, S. Enerback, and B. Lecka-Czernik, "Inducible brown adipose tissue, or beige fat, is anabolic for the skeleton," Endocrinology, vol. 154, no. 8, pp. 2687-2701, 2013.

[22] K. Amrein, S. Amrein, C. Drexler et al., "Sclerostin and its association with physical activity, age, gender, body composition, and bone mineral content in healthy adults," Journal of Clinical Endocrinology and Metabolism, vol. 97, no. 1, pp. 148-154, 2012.

[23] J. C. K. Wells, "Sexual dimorphism of body composition," Best Practice and Research: Clinical Endocrinology and Metabolism, vol. 21, no. 3, pp. 415-430, 2007.

[24] S. R. Davis, K. Z. Walker, and B. J. Strauss, "Effects of estradiol with and without testosterone on body composition and relationships with lipids in postmenopausal women," Menopause, vol. 7, no. 6, pp. 395-401, 2000.

[25] F. S. Mirza, I. D. Padhi, L. G. Raisz, and J. A. Lorenzo, "Serum sclerostin levels negatively correlate with parathyroid hormone levels and free estrogen index in postmenopausal women," Journal of Clinical Endocrinology and Metabolism, vol. 95, no. 4, pp. 1991-1997, 2010.

[26] U. I. Mödder, J. A. Clowes, K. Hoey et al., "Regulation of circulating sclerostin levels by sex steroids in women and in men," Journal of Bone and Mineral Research, vol. 26, no. 1, pp. 27-34, 2011.

[27] N. M. Al-Daghri, K. M. Alkharfy, S. Rahman et al., "Irisin as a predictor of glucose metabolism in children: sexually dimorphic effects," European Journal of Clinical Investigation, vol. 44, no. 2, pp. 119-124, 2014.

[28] M. T. Drake, B. Srinivasan, U. I. Mödder et al., "Effects of parathyroid hormone treatment on circulating sclerostin levels in postmenopausal women," Journal of Clinical Endocrinology and Metabolism, vol. 95, no. 11, pp. 5056-5062, 2010. 


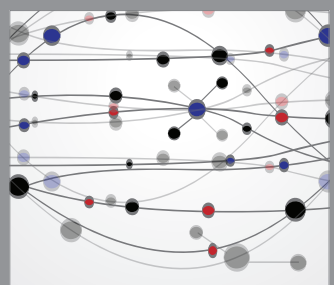

The Scientific World Journal
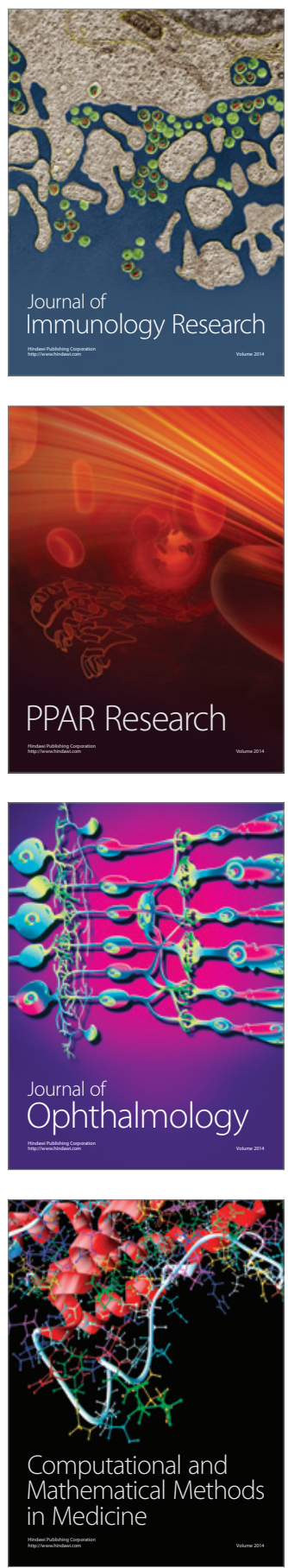

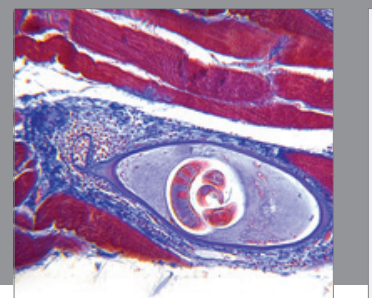

Gastroenterology

Research and Practice
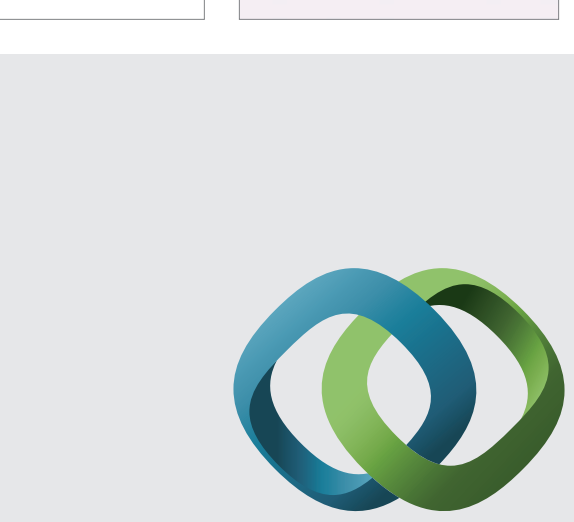

\section{Hindawi}

Submit your manuscripts at

http://www.hindawi.com
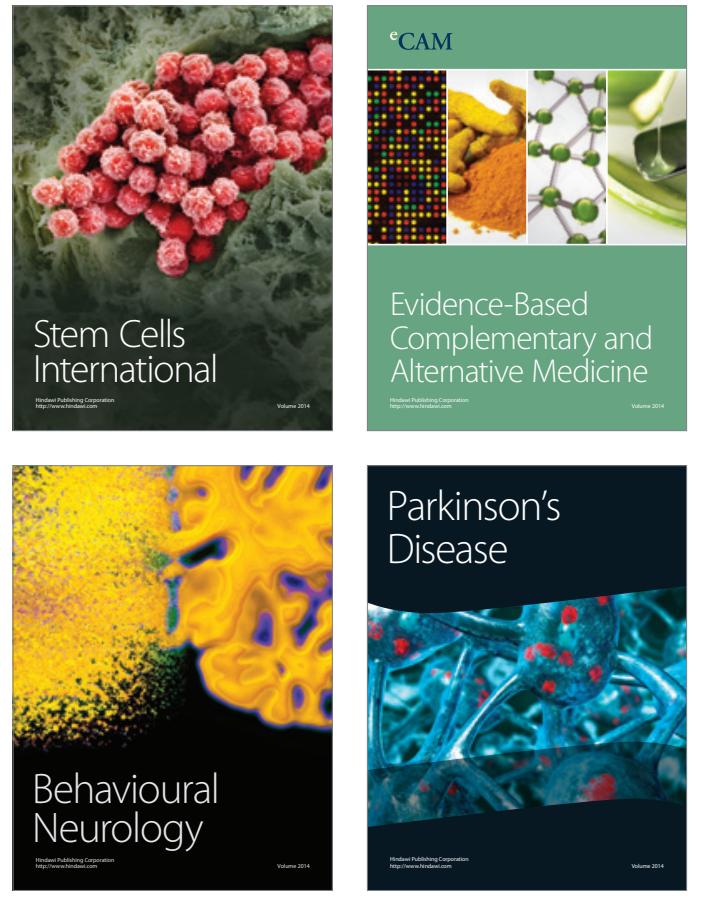
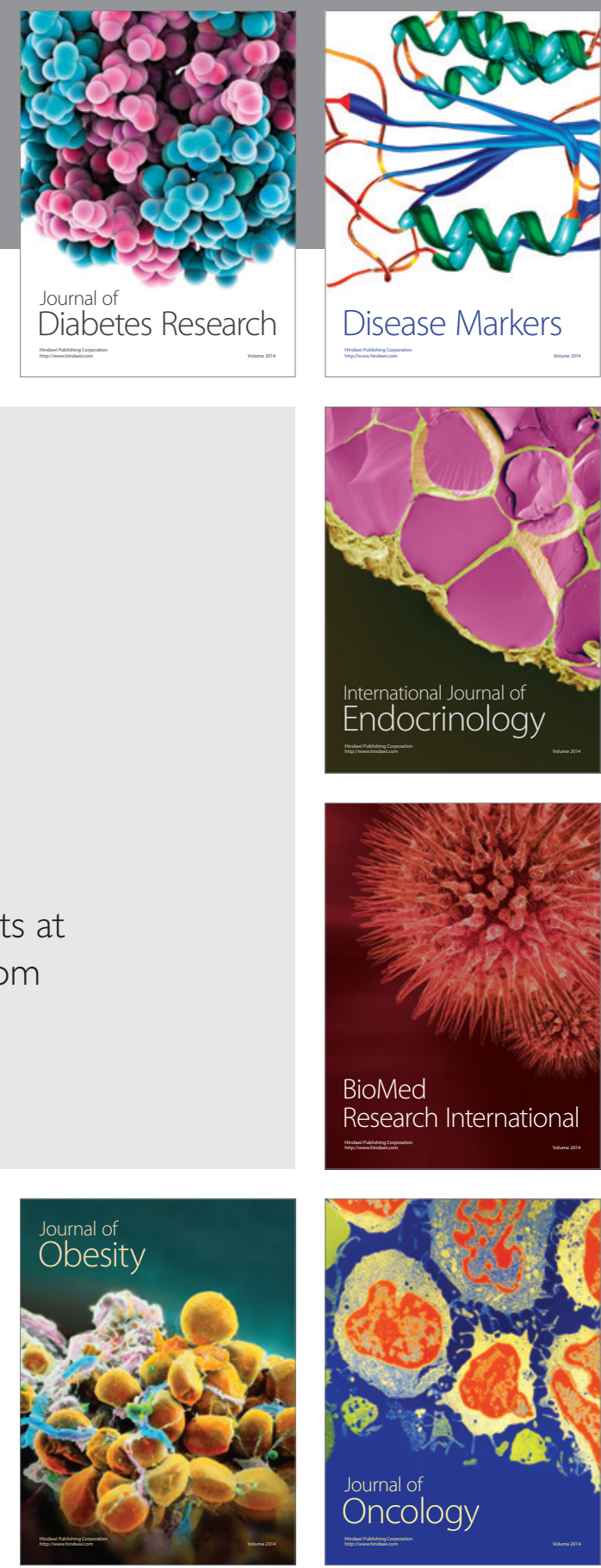

Disease Markers
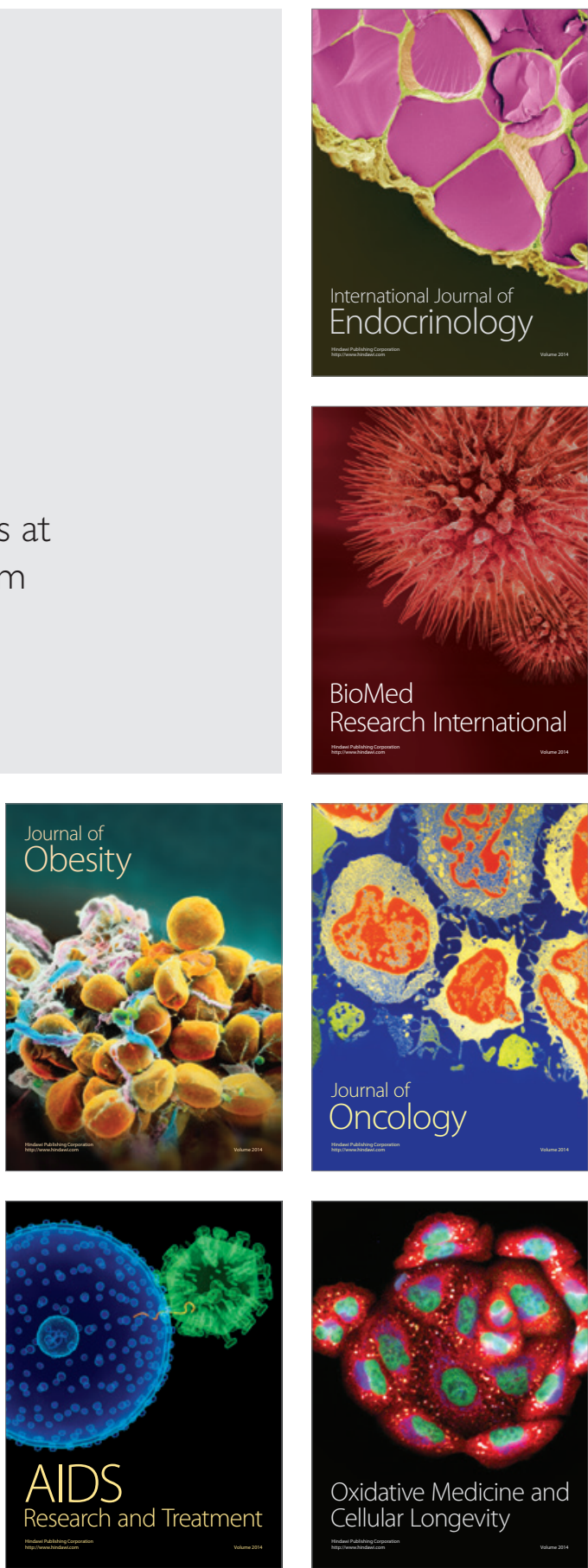